\title{
Recruiting aus Sicht der Bewerber_innen
}

\section{Ein Vergleich klassischer und neuartiger digitaler Methoden im Personalmarketing}

\author{
Meinald T. Thielsch@, Dilara Erdal und Viola Merhof@ \\ Institut für Psychologie, Westfälische Wilhelms-Universität Münster
}

\begin{abstract}
Zusammenfassung: Hochwertiges Personalmarketing ist der essenzielle erste Schritt im Recruiting. Qualifizierte Bewerber_innen müssen auf offene Positionen erfolgreich aufmerksam gemacht werden, wofür Unternehmen und Organisationen verschiedene Online- und OfflineMaßnahmen zur Verfügung stehen. Zentral ist dabei die Wahrnehmung des Personalmarketings durch die Bewerbenden, deren Sicht bislang allerdings wenig betrachtet wurde. In der vorliegenden Studie wird daher mithilfe einer für die deutsche Arbeitsbevölkerung repräsentativen Stichprobe $(N=1.070)$ die Nutzung und Bewertung von klassischen und neuartigen digitalen Personalmarketingmaßnahmen in sozialen Medien untersucht und zusätzlich digitale Ansprachemethoden experimentell überprüft. Bekannte und etablierte Online- und Offline-Personalmarketingmaßnahmen weisen hohe Nutzungszahlen auf und sind zugleich am beliebtesten. Soziale Medien werden hingegen von den Bewerber_innen vergleichsweise wenig präferiert. Kongruent dazu wird im experimentellen Setup die klassische Benachrichtigung per Post neuartigen digitalen Kommunikationswegen (WhatsApp, Facebook, Alexa Voice System) vorgezogen. Die Ergebnisse dieser Studie können in der Praxis zur gezielten Ansprache potenzieller Bewerber_innen und für den optimalen Einsatz verschiedener Methoden genutzt werden.

Schlüsselwörter: Bewerberansprache, Digitalisierung, Employer Branding, E-Recruiting, Personalbeschaffung
\end{abstract}

\begin{abstract}
Recruiting From the Perspective of Applicants: A Comparison of Classical and Novel Digital Methods in Personnel Marketing
Abstract: High-quality personnel marketing is the essential first step in recruiting. Qualified applicants must be successfully made aware of open positions. Central to this is the perception of the personnel marketing activities by the applicants, whose views have not been considered much to date. The aim of this study, therefore, was to examine the use and evaluation of classical and novel digital personnel marketing methods in social media in a representative sample of the German working population $(N=1,070)$. Additional digital methods of addressing applicants were tested experimentally. Well-known and established online and offline personnel marketing methods showed high user rates and were the most popular, whereas addressing applicants via social media was comparatively less preferred by applicants. In the experimental setup, the traditional notification by mail was preferred over novel digital communication channels (i.e., WhatsApp, Facebook, Alexa Voice System). The results of this study can be used in practice to address potential applicants specifically and for the optimal usage of different methods.
\end{abstract}

Keywords: digitization, employer branding, e-recruiting, personnel recruitment, HR marketing

Der Bedarf an Fachkräften in Deutschland war im Jahr 2018 mit 2.7 Millionen Personen so hoch wie noch nie. Davon konnten jedoch nur rund 60 Prozent besetzt werden, über eine Millionen Stellen blieben unbesetzt (Dettmann et al., 2019). Dieser Mangel an Arbeitskräften und der demografische Wandel sorgen dafür, dass es immer weniger Bewerbungen und damit einhergehend größere Konkurrenz um gut ausgebildete Bewerber_innen gibt (Seng, Fiesel, \& Krol, 2012; Weitzel et al., 2015 \& 2020a). Für Unternehmen und Organisationen ist es daher essenziell, möglichst viele potenziell geeignete Bewerber_innen anzusprechen. Eine möglichst hohe Grundquote geeigneter Bewerber_innen ist grundsätzlich für den gesamten nachlaufenden Auswahlprozess von Vorteil, da sich so die Wahrscheinlichkeit erhöht, optimal passende Personen auswählen zu können (Taylor \& Russell, 1939; Thielsch, Träumer, Pytlik, \& Kanning, 2012b). Den Prozess des Recruitings digitalisiert und Internet-basiert zu gestalten ist somit vorteilhaft, da die Ansprache vieler potenzieller Bewerber_innen erleichtert und eine hohe Geschwindigkeit des Bewerbungsprozesses ermöglicht wird (Konradt \& Sarges, 2003; Woods, Ahmed, Nikolaou, Costa, \& Anderson, 2020). Zudem bietet sich die Option, bereits online eine Vorauswahl der Bewerber_innen zu treffen, was Kosten und Zeit spart (Cappelli, 2001). In Anbetracht dieser Vorteile ist ein hohes Interesse an Online-Recrui- 
ting seitens der Unternehmen (Weitzel et al., 2020a), wie auch der Forschung (bspw. Dettmann et al., 2019) nicht verwunderlich.

Allerdings muss bei der Wahl von Recruitingmaßnahmen durch die Unternehmen immer im Vordergrund stehen, wie diese durch die Bewerber_innen genutzt und wahrgenommen werden: Sie bewerten über den gesamten Recruitingprozess hinweg die Attraktivität des Unternehmens und entscheiden letztendlich, ob sie eine Stelle annehmen (Chapman, Uggerslev, Carroll, Piasentin, \& Jones, 2005; Lievens \& Slaughter, 2016). Allerdings gibt es nur wenige Studien, die sich mit der Sicht der Bewerber_innen auf spezifische Maßnahmen befassen (bspw. Thielsch et al., 2012b; Weitzel et al., 2020a) und bislang keine Ergebnisse unter Verwendung einer für die deutsche Arbeitsbevölkerung repräsentativen Stichprobe. Daher mangelt es Unternehmen an einer Entscheidungshilfe, ob und in welchem Ausmaß Online-Maßnahmen im Recruiting eingesetzt werden sollten.

In der vorliegenden Studie soll diese Lücke der aktuellen Forschung geschlossen werden: Es werden Internetbasierte Methoden, inklusive verschiedener sozialer Medien, und klassische Offline-Methoden des Personalmarketings systematisch in Bezug auf die Nutzung und Bewertung durch die Bewerber_innen verglichen und somit ihre Sicht auf den Bewerbungsprozess dargestellt. $\mathrm{Zu}$ sätzlich erfolgt eine experimentelle Prüfung innovativer digitaler Ansprachemethoden im Vergleich zu einer klassischen Benachrichtigung von Bewerber_innen durch ein Unternehmen per Post.

\section{Theoretischer Hintergrund}

Recruiting im Allgemeinen ist von Breaugh (2008) definiert als Maßnahmen, die 1. die Aufmerksamkeit potenzieller Bewerber_innen auf ein Jobangebot lenken, 2. beeinflussen, ob sich diese bewerben, 3. ob das Interesse bis zu einem Jobangebot bestehen bleibt und 4. ob ein Jobangebot angenommen wird. Bislang gibt es keine übergeordnete Theorie, die das Recruiting über diese vier Punkte hinweg beschreibt (Breaugh, 2013). Jedoch ist die Wahrnehmung des gesamten Recruitingprozesses durch die Bewerber_innen in allen Punkten ein entscheidender Prädiktor, ob eine Stelle schlussendlich besetzt werden kann (Chapman et al., 2005; Truxillo, Bauer, \& Garcia, 2017). Es finden sich umfassende Forschungen zum Unternehmens- und Arbeitgeberimage (für eine Übersicht siehe Lievens \& Slaughter, 2016). In der vorliegenden Arbeit sollen allerdings spezifische Recrutingmaßnahmen, insbesondere im Personalmarketing, fokussiert werden.
Der erste, zentrale Schritt des Recruitingprozesses besteht darin, potenzielle Bewerber_innen auf eine zu besetzende Stelle aufmerksam zu machen und Interesse hervorzurufen. Dafür steht Unternehmen eine Vielzahl von Personalmarketingmaßnahmen zur Verfügung, die von klassischen Zeitungsannoncen, über Anzeigen auf der eigenen Website bis hin zu vergleichsweise neuartigen Kommunikationswegen in den sozialen Medien reichen (Thielsch et al., 2012b). Die Internet-basierten Maßnahmen gewinnen seit der Jahrtausendwende zunehmend an Bedeutung (Breaugh, 2013; Chapman \& Gödöllei, 2017). In der Mitte der 2000er Jahre erlebte das World Wide Web eine weitere Innovation: Soziale Medien und die aktive Nutzung von Web 2.0 Angeboten (Busemann \& Gscheidle, 2010). Die Mitgliederzahlen der sozialen Netzwerke stiegen in den Jahren nach deren Einführung rasant an, die Angaben zu den Nutzungszahlen scheinen aber in den letzten Jahren recht vergleichbar: Hinsichtlich einer mindestens wöchentlichen Nutzung gaben im Jahr $201034 \%$ der Befragten an private Netzwerke und Online-Communities $\mathrm{zu}$ verwenden sowie $5 \%$ berufliche Netzwerke (Busemann \& Gscheidle, 2010); im Jahr 2020 waren dies $36 \%$ beziehungsweise $4 \%$ (Beisch \& Schäfer, 2020). Im Gegensatz zu passiven Online-Methoden wie der Stellenanzeige auf der Website sind damit Web 2.0 Anwendungen im Recruiting in Form von sozialen Medien noch vergleichsweise wenig genutzt und daher als eher neuartig zu bezeichnen. Allerdings steigt das Interesse an solchen neuartigen digitalen Methoden wie den sozialen Medien - sowohl in der Fachöffentlichkeit also auch bei Unternehmen (van Iddekinge, Lanivich, Roth, \& Junco, 2016; Weitzel et al., 2020a; Woods et al., 2020). Prominente soziale Medien im eher privaten Bereich sind Facebook, Instagram, und Youtube - mit mindestens wöchentlichen Nutzerzahlen im Bereich von 20 bis $40 \%$ gehören diese zum Alltag vieler Menschen (Beisch \& Schäfer, 2020). Rein berufliche Netzwerke wie Xing und LinkedIn werden von etwa $11 \%$ der Deutschen zumindest gelegentlich genutzt, $4 \%$ geben eine wöchentliche Nutzung an (Beisch \& Schäfer, 2020).

Durch ihre Vielfalt und starke Verzweigung haben die sozialen Medien großes Potenzial, das Recruiting zu revolutionieren (McFarland \& Ployhart, 2015). Die dort verbreiteten Inhalte erreichen breite Nutzergruppen, welche die Informationen wiederum an andere Nutzer weiterleiten können (Taddicken \& Schmidt, 2017). Im Kontext des Personalmarketings ermöglicht diese Dynamik, eine Vielzahl potenzieller Bewerber_innen kosteneffizient und innerhalb kurzer Zeit zu erreichen (Madia, 2011). Zudem sind die sozialen Medien wie auch andere Online-Personalmarketingmaßnahmen nicht nur vorteilhaft in Bezug auf Reichweite und Geschwindigkeit - das Fehlen von Online-Recruiting sowie unattraktiv gestaltete 
Unternehmenswebsites wirken sogar negativ auf das Image des Unternehmens und damit den gesamten Bewerbungsprozess (Göritz \& Moser, 2002; Konradt \& Sarges, 2003; Lyons \& Marler, 2011).

Man könnte daher mutmaßen, dass das Online-Personalmarketing in den sozialen Medien heutzutage den wichtigsten Beitrag zu Neueinstellungen in Unternehmen leistet. Dass dem nicht so ist, zeigen Repräsentativbefragungen von Betrieben in Deutschland durch das Institut für Arbeitsmarkt- und Berufsforschung: Der Anteil an Neueinstellungen, der durch Recruiting in den sozialen Medien zustande kam, lag in den letzten Jahren immer unter $5 \%$ (Bossler, Kubis, \& Moczall, 2017; Brenzel et al., 2016; Kubis, 2019). Andere Online-Verfahren wie Stellenanzeigen auf der Unternehmenswebsite oder in webbasierten Job-Portalen sind hingegen inzwischen klar etabliert und mit jeweils 15\% aller Neueinstellungen deutlich erfolgreicher als die vergleichsweise neuartigen sozialen Medien (Kubis, 2019). Dennoch werden stetig über die letzten Jahre rund die Hälfte der Neueinstellungen über drei klassische Offline-Verfahren vermittelt: Den größten Teil machen die Stellenbesetzungen über persönliche Kontakte mit rund $30 \%$ aus, gefolgt vom Kontakt zur Agentur für Arbeit und Stellenanzeigen in Zeitungen.

Es lässt sich also eine Diskrepanz feststellen: Das Online-Personalmarketing und hier insbesondere die sozialen Medien bringen eine Reihe von Vorteilen mit sich (wie eine erhöhte Reichweite und Zeitersparnis), was sowohl den Bewerber_innen als auch den Unternehmen zugutekommt. Dennoch sind klassische Methoden - offline wie online - immer noch deutlich erfolgreicher und machen einen Großteil der Neubesetzungen aus. Eine mögliche Erklärung liefert die Arbeit von Thielsch et al. (2012b), die sich mit der Wahrnehmung der Personalmarketingmaßnahmen aus Sicht der Bewerber_innen beschäftigt: Die Bewertung der Maßnahmen durch die Bewerber_innen korreliert positiv mit ihrer Bekanntheit, ein Mere-Exposure-Effekt (Zajonc, 1968). Kanning (2016) konnte einen derartigen Mere-Exposure-Effekt auch hinsichtlich der Bewertung verschiedener Auswahlverfahren finden. Möglicherweise werden die sozialen Medien deshalb weniger von den Bewerber_innen genutzt, weil sie vergleichsweise unbekannt und im Recruiting bislang kaum etabliert sind. Dafür spricht die Tatsache, dass nicht generell alle Online-Maßnahmen weniger in tatsächlichen Einstellungen resultieren, sondern dies vor allem für das Recruiting via sozialen Medien gilt (Bossler et al., 2017; Brenzel et al., 2016).

Eine weitere mögliche Ursache für den geringen Erfolg der sozialen Medien besteht darin, dass Unternehmen in stärkerem Ausmaß darauf zurückgreifen, wenn sie Probleme bei der Besetzung von Stellen haben (Arnold, Hillerich-Sigg, \& Nolte, 2017). Wenn somit vornehmlich Un- ternehmen mit Besetzungsproblemen in den sozialen Medien rekrutieren und sich Unternehmen ohne diese Probleme auf andere Methoden verlassen, lässt sich die geringe Erfolgsquote der sozialen Medien erklären. Die Besetzungsprobleme und der Fachkräftemangel sind dabei keineswegs gleich über alle Berufssektoren verteilt (Bechmann et al., 2012; Dettmann et al., 2019), und somit unterscheidet sich auch die Bereitschaft, neuartige Methoden auszuprobieren. Manche Berufssektoren zeigen diese Bereitschaft aber unter Umständen auch deshalb, weil ihre Arbeit traditionsmäßig ohnehin eine starke Internetaffinität mit sich bringt (Thielsch et al., 2012b). Im IT Sektor ist beispielsweise eine verstärkte Nutzung von Online-Maßnahmen zu beobachten (Weitzel et al., 2020a), sodass neue Stellen zum großen Teil durch Anzeigen in Internet-Stellenbörsen oder auf der eigenen Website besetzt werden. Ein ähnliches Muster findet sich aus Sicht der Bewerber_innen, denn auch hier nutzen die ITler vermehrt Online-Methoden bei der Jobsuche (Thielsch et al., 2012b).

Das Personalmarketing wird jedoch nicht nur vom Berufssektor, sondern auch von Merkmalen der zu besetzenden Stelle beeinflusst. Da Unternehmen fast doppelt so viel Zeit für Auswahlgespräche und -tests aufwenden, wenn eine Führungsposition vergeben wird (Arnold et al., 2017), könnte sich eine online-basierte Vergrößerung des Bewerberpools und entsprechende Vorauswahl der geeigneten Bewerber_innen umso mehr lohnen. Dennoch werden Top-Manager bislang wenig über Online-Maßnahmen rekrutiert, beim mittleren Management kommen hingegen die eigene Website oder Online-Portale schon verstärkt zum Einsatz (Gade, Helfritz, \& Murmann, 2018).

Doch für ein erfolgreiches Recruiting - ob für eine Führungsposition oder nicht - reicht allein das Wecken der Aufmerksamkeit geeigneter Bewerber_innen nicht aus. Die Herausforderung besteht darin, das erste Interesse aufrechtzuerhalten, sodass sich die betreffende Person aktiv bewirbt und schlussendlich bereit ist, ein Jobangebot anzunehmen (Breaugh, 2008). Einen besonderen Stellenwert nimmt dabei die Rückmeldung des Unternehmens auf die eingegangene Bewerbung ein, da hier die erste persönliche Interaktion des Unternehmens mit den Bewerber_innen stattfindet (Hausknecht, Day, \& Thomas, 2004). Die Rückmeldung hat einen starken Einfluss auf die Wahrnehmung des Bewerbungsprozesses insgesamt, sowie auf das Erleben von Fairness und Gerechtigkeit (Thielsch, Träumer \& Pytlik, 2012a). Dennoch fokussiert sich die bisherige Forschung stärker auf den Eingang der Bewerbung beim Unternehmen und weniger auf die spätere Rückmeldung bei den Bewerber_innen (Batinic \& Appel, 2009; Weitzel et al., 2016). Dabei ist eine Diskrepanz zwischen den Wünschen von Unternehmen und Bewerber_innen erkennbar: Unternehmen prä- 
ferieren Formularbewerbungen, gefolgt von der Bewerbung per E-Mail und schließlich per Post, wohingegen Bewerber_innen deutlich die E-Mail-basierte Bewerbung bevorzugen (Weitzel et al., 2016). Die digitale Kommunikation zwischen Unternehmen und Bewerber_innen ist für beide Seiten in puncto Zeiteffizienz von Vorteil, aus Sicht der Bewerber_innen spielen aber zusätzliche Faktoren wie die Erfahrung mit Online-Diensten oder Bedenken bezüglich der Privatsphäre eine entscheidende Rolle (Batinic \& Appel, 2009). Denn viele Menschen sind bei der Nutzung des Internets im Allgemeinen (Viseu, Clement, \& Aspinall, 2004) und auch im Bewerbungsprozess (Bohmova \& Malinová, 2013; Woods et al., 2020) um ihre persönlichen Daten besorgt. Beim Einsenden der Bewerbung an das Unternehmen haben Bewerber_innen es bis zu einem gewissen Grad noch selbst in der Hand welche Daten sie digital versenden möchten. Die Rückmeldung des Unternehmens können sie hingegen kaum steuern, sodass die schwierige Abwägung, in welcher Form und in welchem Ausmaß Online-Kommunikationswege im weiteren Bewerbungsprozess eingesetzt werden, bei den Unternehmen liegt.

\section{Fragestellung und Ziel}

Insgesamt stehen Unternehmen und Organisationen vor der Herausforderung, aus einer Vielzahl möglicher Recruitingwege diejenigen zu identifizieren, die kosteneffizient und in kurzer Zeit ermöglichen, Stellen mit geeigneten Kandidat_innen $\mathrm{zu}$ besetzen. Es existiert bislang keine allumfassende Theorie über das bestmögliche Recruiting (Breaugh, 2013). Daher müssen Unternehmen immer wieder neue Wege finden die Wahrnehmung der Organisation durch die Bewerber_innen und damit die Wahrscheinlichkeit einer erfolgreichen Stellenbesetzung positiv zu beeinflussen (Chapman et al., 2005). Die Forschung hängt hier aber der sehr dynamischen Entwicklung gerade der online-basierten Methoden nach (Woods et al., 2020). Es kommt erschwerend hinzu, dass sich die bisherigen Studien zu Personalmarketingmaßnahmen vorrangig auf die Sicht der Unternehmen fokussiert haben (Bossler et al., 2017; Brenzel et al., 2016) und weniger Veröffentlichungen zur Sicht der Bewerber_innen vorliegen (Seng et al., 2012; Thielsch et al., 2012b). Diese Sicht ist aber wichtig: Analysen von Weitzel et al. (2020b) zeigen, dass die Arbeitgeberattraktivität von den Unternehmen selbst positiver eingeschätzt wird als von den Mitarbeiter_innen. Zudem sinkt die Einschätzung der Unternehmensattraktivität nach der Einstellung (Weitzel et al., 2020b). Es besteht somit ein gewisses Risiko, dass Unternehmen ihre eigene Darstellung und die Wirkung be- stimmter Personalmarketingmaßnahmen potenziell $\mathrm{zu}$ positiv einschätzen. Für die Unternehmenssicht liegen zudem Repräsentativerhebungen für Deutschland (z.B. Bossler et al., 2017) bzw. die deutschen Top-1000 Unternehmen (Weitzel et al., 2020a) vor, für die Bewerber_innensicht fehlen diese. Damit mangelt es an einer aussagekräftigen Entscheidungsgrundlage zur Sicht deutscher Arbeitnehmer_innen auf das Personalmarketing. Ziel der vorliegenden Studie ist, diesen Mangel zu beheben und mithilfe einer repräsentativen Stichprobe die Nutzung und Bewertung von verschiedenen Recruitingmaßnahmen durch die Bewerber_innen zu untersuchen.

Dabei werden zwei Aspekte des Bewerbungsprozesses fokussiert: Zum einen die Stellenausschreibung und somit die erste Kontaktaufnahme zu einer breiten Masse an potenziellen Bewerber_innen (Personalmarketingmaßnahmen). Bislang wurden zwar in einzelnen Studien Personalmarketingmaßnahmen aus Sicht der Bewerber_innen untersucht (z.B. Thielsch et al., 2012b; Weitzel et al., 2020a), es fehlt jedoch ein umfassender Vergleich der klassischen mit neuartigen digitalen Maßnahmen mit Fokus auf den sozialen Medien als typische Web 2.0 Anwendung auf Basis einer für die deutsche Arbeitsbevölkerung repräsentativen Stichprobe. Der bisherige Stand der Literatur zeigt eine Diskrepanz auf zwischen erhöhtem Interesse der Unternehmen an neuartigen Internetbasierten Methoden einerseits und geringen Erfolgsquoten andererseits (Brenzel et al., 2016). Daher ist zu vermuten, dass Bewerber_innen eher bekannte, klassische Verfahren präferieren und den sozialen Medien kritisch gegenüberstehen. Ob diese Präferenzen von weiteren Faktoren wie Zugehörigkeit zu einem Berufssektor oder Bekleiden einer Führungsposition beeinflusst werden, soll exploriert werden.

Als zweites Ziel soll die Rückmeldung auf eine beim Unternehmen eingegangene Bewerbung und Einladung zum persönlichen Gespräch mit einem Fokus auf neuartige potenzielle Kommunikationswege untersucht werden (Bewerbungsrückmeldung). Im Vergleich zu einer klassischen Ansprache per Post ist ebenfalls eine kritische Einstellung der Bewerber_innen zu neuartigen Methoden zu vermuten. Wir nehmen an, dass dieses sich insbesondere in Bedenken bezüglich der Privatsphäre begründen (Reinecke \& Trepte, 2008; Viseu et al., 2004; Yao, Rice, \& Wallis, 2007). Ein hohes Bedürfnis nach Privatsphäre sollte mit einer Präferenz für klassische und sicherere Methoden (wie der Ansprache per Post) einhergehen. Zusätzlich werden auch hier mögliche Einflüsse von Berufssektoren oder Führungsverantwortung betrachtet. 
Tabelle 1. Verteilung bezüglich Geschlecht, Alter und Schulabschluss

\begin{tabular}{|c|c|c|c|}
\hline & Eingeladene Stichprobe & Auswertungsstichprobe & Erwerbstätige in Deutschland 2018 \\
\hline \multicolumn{4}{|l|}{ Geschlecht } \\
\hline männlich & 54.00 & 52.99 & 53.42 \\
\hline weiblich & 46.00 & 46.82 & 46.58 \\
\hline Keine/andere Angabe & & 0.19 & \\
\hline \multicolumn{4}{|l|}{ Alter } \\
\hline 18 (20) bis 29 Jahre & 15.00 & 9.07 & 16.59 \\
\hline 30 bis 39 Jahre & 23.00 & 19.91 & 21.01 \\
\hline 40 bis 49 Jahre & 27.00 & 30.75 & 22.09 \\
\hline 50 bis 65 Jahre & 35.00 & 40.19 & 34.68 \\
\hline Keine/andere Angabe & & 0.09 & 5.64 \\
\hline \multicolumn{4}{|l|}{ Schulabschluss } \\
\hline Hauptschulabschluss / Volksschulabschluss & 28.00 & 19.16 & 22.00 \\
\hline Realschulabschluss / polytechnische Oberschule & 38.00 & 38.50 & 35.02 \\
\hline Hochschulreife / Fachhochschulreife & 34.00 & 41.31 & 40.16 \\
\hline kein Schulabschluss oder keine/andere Angabe & & 1.03 & 2.83 \\
\hline
\end{tabular}

Anmerkungen: In der eingeladenen und finalen Stichprobe wurde als Alterskategorie 18 bis 29 Jahre erhoben, bei den Erwerbstätigen in Deutschland handelt es sich um 20 bis 29 Jahre. Die Daten der Erwerbstätigen in Deutschland entstammen einem Bericht des Statistischen Bundesamts (2019). Es bestehen keine statistisch signifikanten Unterschiede in den Verteilungen der Auswertungsstichprobe im Vergleich zur Zusammensetzung der Erwerbstätigen in Deutschland (Geschlecht: $\chi^{2}(2)=0.19, p=.91$; Alter: $\chi^{2}(4)=3.99, p=.41$; Schulabschluss: $\left.\chi^{2}(3)=1.22, p=.75\right)$. Minimale Abweichungen einzelner Prozentwertsummen von 100 ergeben sich durch Rundungseffekte.

\section{Methode}

\section{Stichprobe}

Insgesamt nahmen an der Online-Befragung 1336 Personen teil, von denen jedoch 138 die Umfrage vor ihrem Ende abbrachen und 117 aufgrund offensichtlich unrealer Angaben ${ }^{1}$ oder einer unwahrscheinlich schnellen Bearbeitungsdauer ( $<5$ Minuten für den gesamten Fragebogen) ausgeschlossen wurden. Weitere 11 Befragte wurden ausgeschlossen, da sie angaben, im Vorruhestand zu sein und somit nicht zur Zielgruppe von Recruitingmaßnahmen gehören. Damit umfasste die Stichprobe in der Auswertung 1070 Teilnehmer_innen ${ }^{2}$, von denen 501 weiblich (46.82\%) und 567 männlich (52.99\%) waren, 2 Teilnehmer_innen machten keine Angaben (0.19\%). Das Alter lag im Mittel bei 45.31 Jahren $(S D=10.35)$ und reichte von 18 bis 65 Jahren. Hinsichtlich Geschlecht, Alter und Schulabschluss ist die Auswertungsstichprobe vergleich- bar mit der entsprechenden demographischen Verteilung der deutschen Arbeitsbevölkerung (vgl. Tabelle 1).

Insgesamt 785 Befragte $(73.36 \%)$ gaben an, voll erwerbstätig zu sein. In Tabelle 2 findet sich eine Übersicht zu Tätigkeitsfeldern und den übergeordneten Berufssektoren. Eine Führungsposition hatten $n=270$ Personen (25.23\%) inne; etwa die Hälfte übernahm Verantwortung für bis zu fünf Personen, der Mittelwert lag insgesamt bei $M=19.96(S D=96.92)$. Von allen Befragten hielten $44.67 \%(n=478)$ im Zeitraum von sechs Monaten vor der Befragung Ausschau nach neuen beruflichen Möglichkeiten, $20.37 \%(n=218)$ bewarben sich zum Befragungszeitpunkt aktiv. In den letzten zwei Jahren hatten sich die Befragten auf durchschnittlich 4.77 Stellen beworben $(S D=12.74)$. Zum Zeitpunkt der Befragung wurde das Internet im Durchschnitt seit 16.61 Jahren $(S D=5.34)$ und für durchschnittlich 3.81 Stunden täglich $(S D=2.77)$ genutzt. Der Messenger WhatsApp wurde von $83.27 \%$ $(n=891)$, Facebook von $66.82 \%(n=715)$ und das Alexa

\footnotetext{
Der Ausschluss erfolgte, wenn mindestens an zwei Stellen (von elf Prüfkriterien) auffällige Angaben gemacht wurden. Als auffällige Angabe wurden gewertet: Unreale Altersangaben (< 10 oder >100), die Nutzung von Technologien schon vor ihrer Markeinführung (vier Prüfvariablen zur Technologienutzung von Internet, Facebook, WhatsApp und Alexa; z. B. angegebene WhatsApp-Nutzung seit 15 Jahren, obwohl die Software erst seit 2013 verfügbar ist), eine unwahrscheinlich hohe Anzahl an Bewerbungen für Arbeitsstellen, Weiterbildungsstellen, Praktika oder andere Stellen (jeweils > 100 in den letzten zwei Jahren) und die falsche Beantwortung von Kontrollfragen (insgesamt zwei), deren Antwort im Beschreibungstext vorgegeben wurde.

2 Die Gruppe der Abbrecher und Ausgeschlossenen unterscheidet sich nicht von der nachfolgend verwendeten Stichprobe bezüglich des mittleren Alters oder der Verteilung des Geschlechts. Es unterscheidet sich jedoch der höchste Schulabschluss zwischen beiden Gruppen. Auffällig ist hierbei, dass in der Gruppe der Abbrecher und Ausgeschlossenen weniger Personen die Hochschulreife als Schulabschluss angeben.
} 
Tabelle 2. Berufsfelder und Berufssektoren in der Stichprobe, angelehnt an die Einteilung der Bundesagentur für Arbeit (Matthes, Meinken \& Neuhauser, 2015)

\begin{tabular}{|c|c|c|c|c|c|}
\hline Berufsfeld & $n$ & $\%$ & Berufssektor & $n$ & $\%$ \\
\hline $\begin{array}{l}\text { Unternehmensbezogene Dienstleistungsberufe/ } \\
\text { Beratung }\end{array}$ & 162 & 15.14 & $\begin{array}{l}\text { Kaufmännische und unternehmensbezogene } \\
\text { Dienstleistungsberufe }\end{array}$ & 338 & 31.59 \\
\hline Handelsberufe & 127 & 11.87 & & & \\
\hline Berufe in Unternehmensführung und -organisation & 49 & 4.58 & & & \\
\hline Gesundheitsberufe & 89 & 8.32 & Personenbezogene Dienstleistungsberufe & 231 & 21.59 \\
\hline Soziale und kulturelle Dienstleistungsberufe & 86 & 8.04 & & & \\
\hline Lebensmittel- und Gastgewerbeberufe & 56 & 5.23 & & & \\
\hline Produktionsberufe & 158 & 14.77 & Produktionsberufe & 158 & 14.77 \\
\hline IT- und naturwissenschaftliche Dienstleistungsberufe & 77 & 7.20 & IT- und naturwissenschaftliche Dienstleistungsberufe & 77 & 7.20 \\
\hline Lehrende und ausbildende Berufe & 32 & 2.99 & Lehrende und ausbildende Berufe & 32 & 2.99 \\
\hline Sicherheitsberufe & 32 & 2.99 & Sonstige & 234 & 21.87 \\
\hline Sonstige (z. B. Reinigungsberufe, Logistikberufe) & 200 & 18.69 & & & \\
\hline keine Angaben & 2 & 0.19 & & & \\
\hline
\end{tabular}

Voice System von Amazon von $13.46 \%(n=144)$ der Befragten verwendet.

\section{Vorgehen und Befragungsinstrument}

Zur Studie wurde über einen kommerziellen Felddienstleister eingeladen. Vorgabe war hier eine repräsentative Abbildung der deutschen Arbeitsbevölkerung von 18 bis 65 Jahren in den Merkmalen Geschlecht, Alter und Bildungsgrad. Die Verteilung dieser Merkmale unter allen eingeladenen Teilnehmer_innen ist in Tabelle 1 aufgeführt.

Durch den Klick auf einen Link gelangten die Befragten zur Umfrage, welche in drei Abschnitte gegliedert war (ein vollständiges Codebuch mit allen Itemformulierungen sowie anonymisierte Rohdaten finden sich unter https://doi.org/10.5281/zenodo.4520980). Im ersten Teil wurden nach allgemeinen Hinweisen (zu Studienthema, Verantwortlichen, Kontaktmöglichkeiten, Befragungsdauer, Freiwilligkeit, Anonymität und Datenschutz sowie Zustimmung zur Teilnahme) sowohl demografische Daten als auch Daten zum Mediennutzungsverhalten sowie dem Bewerbungsverhalten erhoben. Die Teilnehmer_innen wurden nach Alter, Geschlecht, ihrem höchsten Schulabschluss, ihrer Erwerbstätigkeit, ihrem Berufsfeld und ihrer Führungsverantwortung mit der Angabe der Personenzahl, für die sie die Verantwortung übernehmen, befragt. Bezüglich des Mediennutzungsverhaltens wurden die Teilnehmer_innen gebeten anzugeben, seit wie vielen Jahren und wie häufig sie das Internet (im Allgemeinen), Facebook, WhatsApp und das Alexa Voice Service von Amazon nutzen. Letzteres ist ein virtueller Assistent, der per Sprachbefehl bedient werden kann.
Außerdem wurde erfragt, ob die Teilnehmer_innen in den letzten sechs Monaten Ausschau nach neuen beruflichen Möglichkeiten gehalten und ob sie sich aktiv beworben hatten. Dabei sollten sie auch angeben, für wie viele Volloder Teilzeitstellen, Praktika oder Nebenjobs, Studiumsoder Weiterbildungsstellen und sonstige Stellen sie sich schätzungsweise in den letzten zwei Jahren beworben hatten.

Im zweiten Teil wurden den Teilnehmer_innen insgesamt 18 Personalmarketingmaßnahmen (siehe Tabelle 3) kurz erläutert, wovon die Hälfte Offline- und die andere Hälfte Online-Maßnahmen darstellen, unter letzteren sieben neuartige Ansprachewege via Web 2.0 / soziale Medien. Ziel war eine breite Zusammenstellung der möglichen Personalmarketingmaßnahmen auf Basis der bisher vorliegenden Studien (Bossler et al., 2017; Brenzel et al., 2016; Thielsch et al., 2012b). Es wurden dabei zum einen klassische Maßnahmen wie Stellenanzeigen in Zeitungen (offline) oder auf Unternehmenswebsites (online) eingeschlossen. Zum anderen wurden eher neuartige oder auch vergleichsweise seltene Maßnahmen wie Kooperationen von Unternehmen zu Lehrern / Dozenten (offline) oder Stellenanzeigen auf Youtube (online, neuartig) verwendet. Es wurden Personalmarketingmaßnahmen fokussiert, die einer offenen Bewerberansprache zuzurechnen sind, damit waren Maßnahmen der internen Personalsuche (z.B. Auswahl aus Praktikant_innen) und die Direktsuche ausgenommen. Ausgenommen waren zudem einzelne seltene, von den Bewerber_innen wenig genutzt und oft eher geschlossene bzw. firmeninterne Maßnahmen wie beispielsweise Planspiele oder Roadshows (siehe Thielsch et al., 2012b). Bei den 18 dargebotenen Personalmarketingmaßnahmen wurde jeweils erfragt, ob diese Maßnahme bekannt ist und bereits genutzt worden war. 
Dafür wurden die vier Kategorien „kenne ich, habe ich aber noch nie genutzt“, „habe ich früher genutzt“, „,nutze ich derzeit" und „kenne ich nicht" verwendet. Diejenigen, die eine Maßnahme kannten, gaben zusätzlich an, wie ansprechend sie diese empfinden. Die fünfstufige LikertSkala reichte von 1 (,spricht mich gar nicht an”) bis 5 (,spricht mich sehr an“).

Im letzten Teil der Befragung erfolgte ein experimenteller within-subject Vergleich. Hier wurden den Teilnehmer_innen randomisiert nacheinander vier fiktive Rückmeldungen zu einer Bewerbung präsentiert. Der Text lautete jeweils: „Sehr geehrte/r Frau/Herr (Ihr Name), vielen Dank für Ihr Interesse an unserem Unternehmen. Wir haben Ihre Bewerbungsunterlagen gesichtet und würden Sie gerne zu einem Vorstellungsgespräch am 20.11.2018, um 14 Uhr einladen. Bitte geben Sie uns doch eine Rückmeldung, ob Sie den Termin wahrnehmen können!" Die Bedingungen Post, Facebook und WhatsApp wurden in einer entsprechenden Optik als Text angezeigt. Für die Bedingung mit dem Alexa Voice Service von Amazon wurde den Befragten eine Audio-Datei vorgespielt, in der der oben genannte Text von dem System vorgelesen wurde.

Anschließend gaben die Teilnehmer_innen jeweils an, ob sie auf die Nachricht antworten würden (ja / nein) und wie sehr sie sich von der Nachricht angesprochen fühlen (von 1 ,spricht mich gar nicht an” bis 5 ,spricht mich sehr an"). Außerdem wurden die Teilnehmer_innen gebeten, die subjektive Wirkung der fiktiven Rückmeldung zu beurteilen. Dafür wurden neun gegensätzliche Wortpaare präsentiert und es sollte je eine der fünf Stufen zwischen den Extremen ausgewählt werden (Bsp. „herkömmlich neuartig"). Diese Abfrage erfolgte angelehnt an den AttrakDiff-Fragebogen zur Testung von Produkten (Hassenzahl, Platz, Burmester, \& Lehner, 2000). Aus diesem wurden sieben inhaltlich passende Adjektivpaare entnommen, ergänzt um die zwei Gegensatzpaare „professionell - unprofessionell“ und „vertrauenswürdig - nicht vertrauenswürdig“.

Im Anschluss wurde die Einstellung zur Privatsphäre anhand der deutschen Übersetzung der CFIP-Skala (Smith, Milberg, \& Burke, 1996) von Harborth und Pape (2018) erhoben. Diese umfasst 15 Items, mit denen die Besorgnis bezüglich der Datensammlung, der unbefugten Weiterverwendung, dem unzulässigen Zugang und der mangelnden Genauigkeit von Informationen erfragt wird. Die globale Einstellung zur Privatsphäre ergibt sich aus dem Mittelwert der vier Skalenwerte. Die Validität der CFIP-Skala wurde von den Autoren umfassend geprüft und die Retest-Reliabilität liegt mit .78 im akzeptablen Bereich.

Am Ende der Befragung wurde den Teilnehmer_innen gedankt, diese erhielten weitere Informationen und hatten die Möglichkeit ihre Angaben aus der Auswertung auszuschließen. Die Studienteilnahme dauerte im Median 12.07 Minuten.

\section{Ergebnisse}

\section{Nutzung und Bewertung der Personalmarketingmaßnahmen}

Die Teilnehmer_innen gaben in der Befragung zu jeder der 18 vorgestellten Personalmarketingmaßnahmen an, inwiefern diese ihnen bekannt war und ob sie diese nutzten. Für die Auswertung wurde dies in drei Kategorien eingeteilt: „,bereits genutzt“ (hier sind frühere und gegenwärtige Nutzung zusammengefasst), „bekannt, nicht genutzt“, und „nicht bekannt“. Zusätzlich wurden die Maßnahmen jeweils von denjenigen, denen sie bekannt war, bewertet. Die Nutzung und Bewertung sind in Tabelle 3 zusammengefasst und nach absteigender Bewertung sortiert.

Die Personalmarketingmaßnahmen unterschieden sich bezüglich ihrer Nutzung signifikant $\left(\chi^{2}(34)=4258.7\right.$, $p<.001)$. Vor allem Stellenanzeigen in Zeitungen, auf Unternehmenswebsites, in Online-Jobportalen, der Kontakt zur Agentur für Arbeit und persönliche Kontakte wurden von einem Großteil genutzt (siehe Tabelle 3). Kaum genutzt wurden Firmenrankings / Auszeichnungen und Stellenanzeigen auf LinkedIn, Facebook, Twitter, Instagram und Youtube. Bei der varianzanalytischen Betrachtung der Bewertung ${ }^{3}$ zeigten sich ebenfalls signifikante Unterschiede zwischen den verschiedenen Personalmarketingmaßnahmen $(F(7.85,2316.83)=97.01$, $\left.p<.001, \eta^{2}=.16\right)$. Am höchsten bewertet wurden persönliche Kontakte, gefolgt von Stellenanzeigen auf Unternehmenswebsites, in Zeitungen und in Online-Jobportalen. Am schlechtesten bewertet wurden Stellenanzeigen in Online-Communities, auf Xing, LinkedIn, Facebook, Youtube, Instagram und Twitter.

Das Ausmaß der Nutzung ging somit einher mit der Bewertung, was sich in einer positiven Rangkorrelation beider Variablen zeigte $\left(r_{S}(13944)=.40, p<.001\right)$. Auffällig ist, dass klassische Maßnahmen (online und offline) vermehrt genutzt und positiv bewertet wurden, wohinge-

\footnotetext{
Für die varianzanalytischen Betrachtungen der Bewertung wurden Alter, Geschlecht und Schulabschluss als Drittvariablen in die Analyse aufgenommen und nur Teilnehmer_innen mit vollständigen Daten berücksichtigt $(n=307)$.
} 
Tabelle 3. Nutzung und Bewertung der Personalmarketingmaßnahmen

\begin{tabular}{|c|c|c|c|c|c|c|}
\hline \multirow[b]{2}{*}{ Personalmarketingmaßnahme } & \multicolumn{3}{|c|}{ Nutzung } & \multicolumn{3}{|c|}{ Bewertung } \\
\hline & $\begin{array}{l}\text { Prozent bereits } \\
\text { genutzt }\end{array}$ & $\begin{array}{l}\text { Prozent bekannt, } \\
\text { nicht genutzt }\end{array}$ & $\begin{array}{l}\text { Prozent nicht } \\
\text { bekannt }\end{array}$ & M & $S D$ & $n$ \\
\hline \multicolumn{7}{|l|}{ Klassische Ansprachewege } \\
\hline Persönliche Kontakte & 54.21 & 35.23 & 10.56 & 4.04 & 0.92 & 957 \\
\hline Stellenanzeigen Unternehmenswebsite & 54.95 & 33.74 & 11.31 & 3.70 & 1.07 & 949 \\
\hline Stellenanzeigen Zeitungen/ Zeitschriften & 71.40 & 24.49 & 4.11 & 3.62 & 1.00 & 1026 \\
\hline Stellenanzeigen Online-Jobportale & 54.02 & 34.77 & 11.21 & 3.51 & 1.08 & 950 \\
\hline $\begin{array}{l}\text { Kontakte von Unternehmen zu Lehrern/ } \\
\text { Dozenten }\end{array}$ & 22.06 & 47.94 & 30.00 & 3.00 & 1.10 & 749 \\
\hline (Inhouse) Bewerbertage & 22.06 & 51.31 & 26.64 & 3.00 & 1.09 & 785 \\
\hline Karrieremessen/ Personalmessen & 28.32 & 51.50 & 20.19 & 2.90 & 1.11 & 854 \\
\hline Vorträge/ Firmenpräsentationen & 21.40 & 53.27 & 25.33 & 2.89 & 1.07 & 799 \\
\hline Karrierebroschüren/Imageanzeigen & 26.07 & 50.84 & 23.08 & 2.84 & 1.07 & 823 \\
\hline Firmenrankings/ Auszeichnungen & 16.17 & 48.50 & 35.33 & 2.75 & 1.08 & 692 \\
\hline Kontakt zur Agentur für Arbeit & 56.26 & 38.22 & 5.51 & 2.71 & 1.13 & 1011 \\
\hline \multicolumn{7}{|l|}{ Neuartige Ansprachewege } \\
\hline Stellenanzeigen auf Xing & 22.62 & 47.94 & 29.44 & 2.70 & 1.22 & 755 \\
\hline Stellenangebote Online-Communities & 24.86 & 46.26 & 28.88 & 2.56 & 1.13 & 761 \\
\hline Stellenanzeigen auf Linkedln & 16.54 & 44.86 & 38.60 & 2.50 & 1.20 & 657 \\
\hline Stellenanzeigen auf Facebook & 17.76 & 46.73 & 35.51 & 2.42 & 1.19 & 690 \\
\hline Stellenanzeigen auf Youtube & 7.57 & 38.41 & 54.02 & 2.15 & 1.11 & 492 \\
\hline Stellenanzeigen auf Instagram & 7.66 & 38.60 & 53.74 & 2.12 & 1.10 & 495 \\
\hline Stellenanzeigen auf Twitter & 7.85 & 38.97 & 53.18 & 2.07 & 1.07 & 501 \\
\hline
\end{tabular}

Anmerkungen: Die Fragen bezüglich der Nutzung wurden von allen Teilnehmer_innen beantwortet ( $n=1$ 070). Die Bewertungsskala reichte von 1 (,spricht mich gar nicht an“) bis 5 („spricht mich sehr an“). Minimale Abweichungen einzelner Prozentwertsummen von 100 ergeben sich durch Rundungseffekte.

gen neuartige digitale Maßnahmen (wie bspw. Facebook und Youtube) durchweg schlechter abschnitten.

Weiterhin fanden sich für einige der Personalmarketingmaßnahmen (Stellenanzeigen auf Unternehmenswebsite, in Online-Communities, auf Xing, auf LinkedIn, Vorträge / Firmenpräsentationen, Firmenrankings / Auszeichnungen) bezüglich der Nutzung signifikante Unterschiede zwischen den Berufssektoren (mit Bonferroni Korrektur für multiples Testen, $29.22 \leq \chi^{2} \leq 48.30, d f=10$, $p<.05)$. Für alle anderen Maßnahmen zeigten sich keine Unterschiede in der Nutzung $\left(10.28 \leq \chi^{2} \leq 25.90, d f=10\right.$, $p>$.05). Auffällig ist, dass Personen aus dem IT- und naturwissenschaftlichen Sektor bei vielen Maßnahmen ein höheres Nutzungsmaß angaben als Personen aller anderer Berufssektoren. Die Nutzung der Maßnahmen unterteilt nach Berufssektoren ist im Online-Supplement https://doi.org/10.5281/zenodo.4520980 dargestellt.

Bezüglich der Bewertung der Personalmarketingmaßnahmen zeigten sich keine allgemeinen Unterschiede zwischen den Berufssektoren $(F(5,295)=0.48, p=.79)$, aber eine signifikante Interaktion mit den Personalmarketingmaßnahmen $(F(39.27,2316.83)=1.46, p<.05$, $\left.\eta^{2}=.01\right)$. Es wird jedoch deutlich, dass die Unterschiede zwischen den Maßnahmen (mit einer Effektstärke $\eta^{2}=.16$ ) weitaus größer waren als die Abweichungen einzelner Berufssektoren $\left(\eta^{2}=.01\right)$. Beliebte und von vielen Befragten genutzte Maßnahmen zeigten sich relativ unabhängig vom Berufssektor, das heißt Stellenanzeigen in Zeitungen, auf Unternehmenswebsites, in Online-Jobportalen und persönliche Kontakte wurden in verschiedenen Sektoren gleichermaßen genutzt und bewertet.

Zwischen Personen mit und ohne Führungsverantwortung zeigten sich für fast alle Personalmarketingmaßnahmen signifikante Unterschiede in der Nutzung, ebenfalls kontrolliert für multiples Testen $\left(23.31 \leq \chi^{2} \leq 71.52, d f=2\right.$, $p<.001)$. Einzig die Stellenanzeigen auf der Unternehmenswebsite, in Zeitungen und in Online-Jobportalen, sowie der Kontakt zur Agentur für Arbeit unterscheiden sich hier nicht $\left(3.28 \leq \chi^{2} \leq 11.15, d f=2, p>.05\right)$. Insgesamt gaben mehr Personen mit Führungsverantwortung an, die jeweiligen Personalmarketingmaßnahmen $\mathrm{zu}$ nutzen (derzeit oder früher) als Personen ohne Führungsverantwortung (eine detaillierte Darstellung findet sich im Online-Supplement unter https://doi.org/10.5281/zenodo. 4520980). Die Ergebnisse der varianzanalytischen Auswertung der Bewertung deckten sich mit diesem Bild: 
Personen mit Führungsverantwortung bewerteten die Maßnahmen insgesamt positiver $(F(1,295)=16.52$, $\left.p<.001, \eta^{2}=.02\right)$, mit einer im Durchschnitt um 0.43 Punkten höheren Bewertung. Auch hier wird durch die Betrachtung der Effektstärken jedoch deutlich, dass die Unterschiede zwischen den Maßnahmen $\left(\eta^{2}=.16\right)$ größer waren als der Einfluss der Führungsverantwortung.

Hinsichtlich weiterer Teilgruppen fanden sich vereinzelt signifikante Korrelationen, insbesondere hinsichtlich Alter und Schulabschluss (siehe Online-Supplement unter https://doi.org/10.5281/zenodo.4520980). Hierzu waren keine Hypothesen formuliert, dennoch sollen potenzielle Unterschiede kurz exploriert werden. Dafür wurden die Teilnehmer_innen anhand des Medians des Alters (47) in eine jüngere und ältere Gruppe aufgeteilt, Schulabschluss wurde dreigestuft betrachtet. Lediglich bei Stellenanzeigen in Zeitungen $\left(X^{2}(2)=4.92, p=1\right.$ nach Bonferroni Korrektur) und bei Karrierebroschüren / Imageanzeigen $\left(X^{2}(2)=7.30, p=.47\right.$ nach Bonferroni Korrektur) zeigte sich eine ähnliche Nutzung von jüngeren und älteren Personen. Für alle anderen Personalmarketingmaßnahmen ergaben sich signifikante Unterschiede $\left(13.30 \leq \chi^{2} \leq 36.05, d f=2, p<.05\right)$, wobei für alle Maßnahmen in der Gruppe Jüngerer mehr Teilnehmer_innen eine Nutzung angaben als in der Gruppe älterer Teilnehmer_innen. Der Einfluss des Alters auf die Nutzung kann jedoch die zuvor dargestellten Unterschiede zwischen Berufssektoren nicht erklären, da es keine Altersunterschiede zwischen diesen Gruppen gab $(F(5,1061)=1.80$, $p=.11)$. Ebenso unterschied sich das Alter nicht zwischen Personen mit und ohne Führungsverantwortung ( $t(1067)$ $=1.05, p=.30)$. Ein ähnliches Bild zeigte sich hinsichtlich des Schulabschlusses: Keine Unterschiede ergaben sich bei Stellenanzeigen in Zeitungen, dem Kontakt zur Agentur für Arbeit sowie Stellenanzeigen via Facebook, Youtube, Instagram und Twitter $\left(2.64 \leq \chi^{2} \leq 5.64, d f=4\right.$, $p>$.05). Bei allen anderen Maßnahmen zeigte sich bei Befragten mit (Fach)Hochschulreife eine signifikant höhere Nutzung $\left(24.73 \leq \chi^{2} \leq 67.62, d f=4, p<.01\right)$. Unterschiede in der Bewertung korrespondieren hierzu, fallen jedoch zumeist klein aus (Tabellen und Abbildungen dazu im Online-Supplement unter https://doi.org/10.5281/zen odo.4520980).

\section{Bewerbungsrückmeldung}

Im experimentellen Teil der Studie wurden den Teilnehmer_innen randomisiert nacheinander vier fiktive Rück- meldungen (via Post, WhatsApp, Facebook und Alexa) zu einer Bewerbung präsentiert, in der zu einem Vorstellungsgespräch eingeladen wurde. In Tabelle 4 sind die Bewertungen und die jeweiligen Anteile der Teilnehmer_innen, die auf diese Rückmeldung antworten würden, dargestellt.

Es gab signifikante Unterschiede in der Bewertungen der Rückmeldungen ${ }^{4}(F(2.84,2902.46)=406.50$, $\left.p<.001, \eta^{2}=.18\right)$. Post-hoc Tests mit Bonferroni Korrektur zeigten, dass sich alle vier Rückmeldungen in der Bewertung jeweils voneinander unterschieden $(6.30 \leq t$ $\leq 31.97, d f=1033, p<.001)$. Es gab keinen Einfluss des Berufssektors auf die Bewertung der Rückmeldungen, weder als Haupteffekt $(F(5,1022)=0.98, p=.43)$, noch als Interaktion mit der Rückmeldungsart $(F(14.20$, $2902.46)=1.08, p=.37)$. Der Einfluss von Führungsverantwortung war hingegen signifikant $(F(1,1022)=6.76$, $p<.01)$, wie auch in Interaktion mit der Art der Rückmeldung $(F(2.84,2902.46)=1.67, p<.05)$. Post-hoc Tests mit Bonferroni Korrektur zeigten, dass Personen mit im Vergleich zu ohne Führungsverantwortung die Rückmeldung via Alexa als positiver bewerteten $(t(408.83)=3.19$, $p<.01, d=0.22)$, wobei dieser Effekt laut Konvention gering war. Für die anderen drei Rückmeldungen per Post $(t(483.04)=0.58, p=1)$, WhatsApp $(t(463.27)=1.54$, $p=.50)$ und Facebook $(t(460.22)=1.28, p=.80)$, gab es keinen Unterschied je nach Führungsverantwortung.

Tabelle 4. Bewertung der fiktiven Bewerbungsrückmeldungen

\begin{tabular}{lccc}
\hline & Prozent Antwort & Bewertung M & Bewertung SD \\
\hline Post & 96.16 & 3.89 & 0.93 \\
WhatsApp & 78.22 & 3.05 & 1.24 \\
Facebook & 69.25 & 2.81 & 1.28 \\
Alexa & 64.13 & 2.42 & 1.26 \\
\hline
\end{tabular}

Anmerkungen: Die Bewertungsskala reichte von 1 (.,spricht mich gar nicht an") bis 5 (.,spricht mich sehr an“). N = 1070 für Post, WhatsApp und Facebook, $n=1037$ für Alexa. „Prozent Antwort“ bezieht sich auf den Anteil der Befragten die angaben, sie würden auf diese Form der Bewerbungsrückmeldung entsprechend reagieren und dem Unternehmen antworten.

Ein zentraler angenommener Einflussfaktor auf die Wahrnehmung der Bewerbungsrückmeldung ist das Bedürfnis nach Privatsphäre. Dieses wurde durch die CFIPSkala (Smith et al., 1996) operationalisiert und lag im Mittel bei $5.77(S D=0.92)$. Hohe Werte drücken eine hohe Besorgnis bezüglich der Datensammlung, der unbefugten Weiterverwendung, dem unzulässigen Zugang und

\footnotetext{
Für die varianzanalytischen Betrachtungen der Bewertung wurden Alter, Geschlecht und Schulabschluss als Drittvariablen in die Analyse aufgenommen und nur Teilnehmer_innen mit vollständigen Daten berücksichtigt ( $n=1067)$.
} 


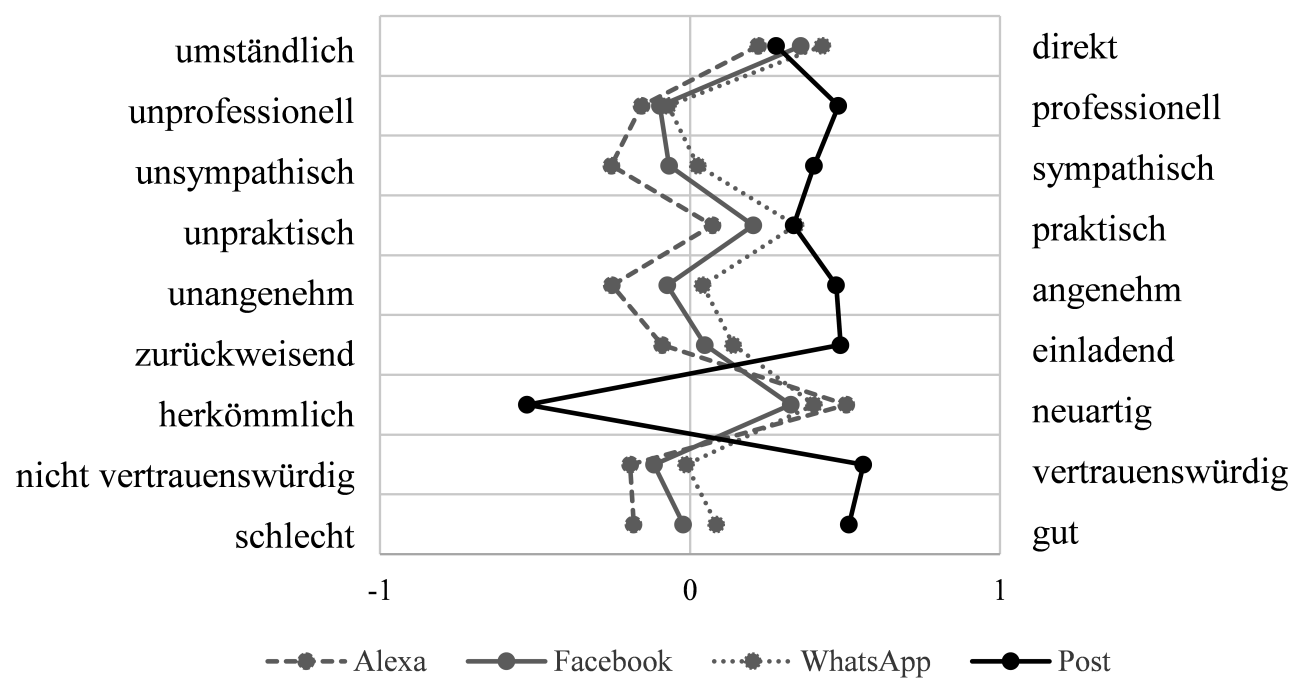

Anmerkungen: Dargestellt sind die mittleren Antworten über N = 1070 Teilnehmer_innen. Ein Wert von 0 drückt dabei eine neutrale Einschätzung genau zwischen beiden Extremen aus, die Werte 1 bzw. -1 entsprechen den Extremen. Das positive Wort jeden Paares ist auf der rechten Seite abgetragen, sodass Mittelwerte >0 eine durchschnittlich positivere Wirkung anzeigen. Sieben der neun Items entstammen dem AttrakDiff-Fragebogen (Hassenzahl et al., 2000), zwei weitere (.,professionell - unprofessionell“ und „vertrauenswürdig - nicht vertrauenswürdig“) wurden ergänzt. Die Unterschiede in der Bewertung sind für alle vier Arten der Rückmeldung in Bezug auf alle neun Items signifikant unterschiedlich, bis auf drei Ausnahmen (Item „direkt“: Post vs. Alexa, Item „professionell“: Facebook vs. WhatsApp sowie Item „praktisch“: Post vs. WhatsApp).

Abbildung 1. Subjektive Wirkung der vier fiktiven Bewerbungsrückmeldungen.

der mangelnden Genauigkeit von Informationen aus. Das Bedürfnis nach Privatsphäre korrelierte leicht positiv mit der Bewertung der Post-Bedingung $(r(1068)=.19$, $p<.001)$, sodass diese Art der Rückmeldung von Personen mit hohem Bedürfnis nach Privatsphäre positiver bewertet wurde. Für WhatsApp $(r(1068)=-.09, p<.01)$, Facebook $(r(1068)=-.15, p<.001)$ und Alexa $(r(1035)=-.11, p<.001)$ ergab sich ein umgekehrter Effekt und leicht negative Korrelation.

Nach jeder Rückmeldung gaben die Teilnehmer_innen ihren subjektiven Eindruck an, welcher durch neun an den AttrakDiff-Fragebogen (Hassenzahl et al., 2000) angelehnten bipolaren Items erfragt wurde. Die Ergebnisse sind in Abbildung 1 visualisiert. Rein deskriptiv ist eine hohe Ähnlichkeit der drei neuartigen Rückmeldungsmethoden und deutliche Abweichungen zur Post-Bedingung erkennbar. Auffällig ist, dass die Post bei den meisten Adjektivpaaren besser abschnitt, jedoch erwartungsgemäß als herkömmlich und wenig neuartig beschrieben wurde.

\section{Diskussion}

Besonders in Zeiten des Fachkräftemangels und großer Konkurrenz um geeignete Bewerber_innen, ist für Unternehmen ein gezieltes Personalmarketing essenziell (Dettmann et al., 2019; Seng et al., 2012). Die vorliegenden
Studie liefert hier ein klares Bild auf Basis einer für die deutsche Arbeitsbevölkerung repräsentativen Stichprobe: Von der Mehrheit der Befragten wird angegeben Stellenanzeigen in Zeitungen, auf Unternehmenswebsites, in Online-Jobportalen, persönliche Kontakte und der Kontakt zur Agentur für Arbeit bereits genutzt zu haben. Bis auf Letzteres entsprechen diese genau den Maßnahmen, die am höchsten bewertet werden und stellen somit aus Sicht der Bewerber_innen die beliebtesten Ansprachewege dar. Auffällig ist, dass es sich dabei durchweg um klassische Verfahren handelt, die entweder offline stattfinden oder sich schon seit langem online etabliert haben. Neuartige digitale Web 2.0 Maßnahmen über soziale Medien (Stellenanzeigen in Online-Communities, auf Xing, LinkedIn, Facebook, Youtube, Instagram und Twitter) werden hingegen von vergleichsweise wenigen Personen genutzt und am schlechtesten bewertet. Damit findet sich ein Mere-Exposure-Effekt in der Bewertung von Personalmarketingmaßnahmen. Die Höhe der hier vorliegenden Rangkorrelation $(r=.4)$ ist vergleichbar mit gefundenen Mere-Exposure-Korrelationen in der Bewertung von Personalauswahlverfahren (Kanning, 2016).

In der vorliegenden Studie bestätigt sich somit das bisherige Bild der Studienlage hinsichtlich der Bedeutung von Stellenanzeigen auf der Unternehmenswebsite, in Online-Jobportalen und Zeitungen (Bossler et al., 2017; Thielsch et al., 2012b), wobei letztere deutlich stärker genannt werden als in der auf die Top-1000 Unternehmen fokussierte Erhebung von Weitzel et al. (2020a). Die Be- 
deutung klassischer Zeitungsanzeigen sowie insbesondere die klar positive Bewertung von persönlichen Kontakten im Personalmarketing aus Sicht der Bewerber_innen in der aktuellen Studie passt zu in Bossler et al. (2017) berichteten Nutzung durch Unternehmen und entsprechenden Erfolgsquoten. Die etablierten Online- und Offline-Maßnahmen haben in den letzten Jahren stetig den größten Beitrag zu Neueinstellungen in Unternehmen geleistet (Bossler et al., 2017; Brenzel et al., 2016; Kubis, 2019), soziale Medien spielen hier bislang nur eine geringe Rolle (Bossler et al., 2017; Brenzel et al., 2016; Kubis, 2019; Weitzel et al., 2020a). Die beruflich ausgerichteten sozialen Medien wie Xing und LinkedIn schneiden dabei bei den Befragten relativ gesehen besser ab als die vornehmlich privat genutzten Netzwerke wie Facebook. Ob die sozialen Medien deswegen relativ schlecht bewertet werden, weil sie sich noch nicht etablieren konnten oder ob es andere Gründe gibt, kann aufgrund dieser Studie nicht beantwortet werden. Kongruent zur bisherigen Literatur (Thielsch et al., 2012b; Weitzel et al., 2020a) werden die sozialen Medien jedoch vom IT Sektor bereits stärker genutzt, verglichen mit anderen Berufssektoren. $\mathrm{Zu}$ beachten ist allerdings, dass die Unterschiede zwischen den Berufssektoren weitaus geringer sind als zwischen den Personalmarketingmaßnahmen, sodass die oben genannten klassischen Maßnahmen grundsätzlich von mehr ITlern genutzt und präferiert werden. Ähnliches gilt für den Einfluss der Führungsverantwortung: Zwar werden die meisten Maßnahmen von mehr Personen in Führungspositionen genutzt und besser bewertet, die Effekte sind aber relativ gering, sodass hieraus keine Handlungsempfehlungen abgeleitet werden können.

Im Mittelfeld der Beliebtheit liegt eine breite Palette von reinen Offline-Maßnahmen, angefangen bei Kooperationen von Unternehmen zu Lehrern / Dozenten über Karrieremessen bis hin zum Kontakt zur Agentur für Arbeit (siehe Tabelle 3). Obwohl es sich bei den meisten dieser Maßnahmen nicht um Klassiker des Personalmarketings handelt, werden sie durchaus von einigen Bewerber_innen genutzt und positiv bewertet. Vor allem die Stellenveröffentlichung bei der Agentur für Arbeit, die sich bereits als erfolgreich bezüglich Neueinstellungen erwiesen hat (bspw. Kubis, 2019), zeigt auch in dieser Studie hohe Nutzungsquoten seitens der Bewerber_innen (die Bewertung ist jedoch nur knapp im mittleren Bereich).

Da die Anziehung der Aufmerksamkeit potenzieller Bewerber_innen nur den ersten Schritt im Recruiting darstellt, sollte in der vorliegenden Studie als weiteres Ele- ment die fiktive Rückmeldung zu einer eingegangenen Bewerbung untersucht werden. Die klassische Rückmeldung per Post zeigt sich gegenüber sehr neuartigen Antwortwegen überlegen, was sich auch in einer hohen Antwortbereitschaft widerspiegelt. Weniger beliebt ist eine Rückmeldung mittels innovativer digitaler Wege - also per WhatsApp, gefolgt von Facebook und schlussendlich Alexa. Es findet sich somit wiederum, dass vergleichsweise neuartige Online-Maßnahmen nicht mit klassischen Wegen mithalten können, was die Bewertung durch potenzielle Bewerber_innen angeht. Da es keine Unterschiede zwischen Berufssektoren und nur kleine Effekte bei der Führungsverantwortung gibt, ist für alle Bewerbergruppen die Rückmeldung auf einem klassischen Weg $\mathrm{zu}$ empfehlen. ${ }^{5}$ Unterstützt wird dieser Eindruck durch die subjektiven Einschätzungen ermittelt durch neun Adjektivpaare: Die Rückmeldung per Post wird in fast allen Kategorien (ausgenommen der Neuartigkeit) gut bis sehr gut bewertet und hebt sich von neuartigen Wegen der Rückmeldung ab. So wird die Post als vertrauenswürdig eingeschätzt, wohingegen die Rückmeldungen per WhatsApp, Facebook oder Alexa neutrale bis niedrige Werte zeigen. Übereinstimmend dazu ist das Bedürfnis nach Privatsphäre positiv mit der Bewertung der postalischen Rückmeldung und negativ mit den drei neuartigen Online-Rückmeldungen korreliert. Zwar ist eine Aussage zur Kausalität nicht möglich, es ist jedoch zu vermuten, dass die Besorgnis um persönliche Daten im Bewerbungsprozess eine erhöhte Präferenz der analogen Rückmeldung per Post zur Folge haben kann (Reinecke \& Trepte, 2008; Viseu et al., 2004; Yao et al., 2007). Ähnliche Sorgen hinsichtlich der Privatsphäre finden sich auch in Studien zu anderen neuartigen digitalen Formen in der Personalauswahl, wie bspw. automatisierten Interviews (Langer, König \& Krause, 2017; Langer, König \& Papathanasiou, 2019). Allerdings klärt das Bedürfnis nach Privatsphäre nur einen Teil der Varianz auf, sodass dies vermutlich nur einer der Gründe für die höheren Bewertungen der Post Bedingung ist. Möglicherweise gilt auch hier, dass sich neue Methoden zunächst stärker im Alltag etablieren müssen, ehe sie für persönlich bedeutsame Prozesse wie Bewerbungen verwendet werden. Denn trotz der steigenden Präsenz von Online-Diensten im beruflichen und privaten Umfeld (Beisch \& Schäfer, 2020; Seng et al., 2012), nutzt fast jeder Fünfte der hier verwendeten Stichprobe kein WhatsApp, jeder Dritte kein Facebook und das Alexa Voice System wird sogar nur von rund $13 \%$ verwendet.

\footnotetext{
Zu den klassischen Rückmeldewegen zählt aus Sicht der Autoren neben der getesteten Postvariante auch das persönliche Gespräch (auch via Telefon) oder der Post ähnliche Rückmeldewege wie E-Mail. Bei digitaler Kommunikation sind hierbei Aspekte der Datensicherheit zu beachten.
} 


\section{Limitation und Implikationen für zukünftige Forschung}

Die vorliegenden Ergebnisse sollten unter Einbezug der Limitationen der Studie interpretiert werden. Gleichzeitig eröffnen sich hier jedoch an vielen Stellen direkte Ansatzpunkte für zukünftige Forschungen. $\mathrm{Zu}$ bedenken ist zunächst, dass die verschiedenen Personalmarketingmaßnahmen nicht für jedes Unternehmen gleichermaßen sinnvoll sind. Beispielsweise nutzen einige kleinere Unternehmen aufgrund von hohen Kosten keine Stellenanzeigen in Zeitungen (Dietz, Kubis, Leber, Müller, \& Stegmaier, 2013) und verwenden beispielsweise kostengünstige Plakate oder Aushänge im Schaufenster. Zudem färbt die Art des Personalmarketings auf das Image des Unternehmens ab (Allen, Mahto, \& Otondo, 2007; Girard \& Fallery, 2011), sodass ein junges Startup, bei dem man sich nicht über die sozialen Medien bewerben kann, möglicherweise wenig attraktiv erscheint. In der vorliegenden Studie konnten Unternehmensgröße und -kultur und entsprechende individuell zugeschnittene Maßnahmen (wie Plakate) nicht spezifisch untersucht werden. Eine gezielte Betrachtung spezifischer Unternehmenstypen oder Brancheneigenschaften ist hier ein lohnenswerter Ansatz für zukünftige angewandte Studien. Gleiches gilt für die möglichen direkten Rückmeldewege an die Bewerber_innen: In der vorliegenden Studie war das Ziel ein Kontrast zwischen sehr klassischem (Post) und sehr neuartigen Wegen (Facebook, WhatsApp, Alexa). Aus zeitlichen Gründen war hierbei nicht möglich ein umfassendes Spektrum an Kommunikationswegen abzubilden. $\mathrm{Zu}-$ künftige Studien könnten dies fokussieren und weitere klassische (E-Mail, Telefon) wie auch neuartige Wege (Video-Call, App-basierte Lösungen) gezielt untersuchen. Insbesondere die Betrachtung dieser verschiedenen Antwortformate in Hinblick auf das Bedürfnis nach Privatsphäre könnten dabei interessant sein. Nicht zuletzt könnte dieser Aspekt ebenso wie die generelle Akzeptanz von aktiven Rekrutierungswegen (z. B. Direktsuche, Arbeitsvermittlung, Ansprache via Bewerberpools) in zukünftigen Studien fokussiert werden.

Eine weitere Limitation der vorliegenden Studie ist, dass nur der Kommunikationsweg einer Stellenanzeige allgemein untersucht wurde, jedoch nicht ihr vermittelter Inhalt im Detail, welcher einen entscheidenden Beitrag im Recruiting leistet. Denn ein hoher wahrgenommener Person-Job Fit wirkt sich mediiert über die Unternehmensattraktivität positiv auf die Wahrscheinlichkeit aus, dass eine Stelle angenommen wird (Carless, 2005). Allerdings ist die Informationsdichte nicht bei allen Personalmarketingmaßnahmen gleichermaßen hoch, da Unternehmen bspw. auf ihrer eigenen Website so umfang- reiche Informationen $\mathrm{zu}$ einer ausgeschriebenen Stelle vermitteln können, wie bei kaum einer anderen Maßnahme. Außerdem erscheint es unpassend, eine Stellenanzeige in der Zeitung mit demselben Wortlaut in den sozialen Medien zu veröffentlichen, da Sprachstil und Umfang von Nachrichten sich im Allgemeinen stark unterscheiden (Storrer, 2013). Möglicherweise bewerteten die Teilnehmer_innen der Studie somit nicht nur die Maßnahme an sich, sondern unbewusst auch ihrer früheren Erfahrungen über die jeweilige Art der Informationsübermittlung. An dieser Stelle lohnt sich zudem zukünftig die gleichzeitige Betrachtung sowohl der Unternehmens- als auch der Bewerberinnenseite. Weitzel et al. (2020b) konnten aufzeigen, dass Unternehmen tendenziell ihre eigene Arbeitgeberattraktivität überschätzen - und dieser Wert nach der Einstellung bei den Mitarbeitenden noch einmal sinkt. Hier wäre ein Abgleich der Perspektiven auf die inhaltliche Gestaltung des Personalmarketings (insbesondere in Hinblick auf eine reale Tätigkeitsvorschau, siehe Breaugh, 2013) vielversprechend.

Hinsichtlich der ausgewerteten Stichprobe ist zu bedenken, dass Personen im Alter von 18 bis 30 Jahren zwar nicht signifikant aber dennoch nummerisch leicht unterrepräsentiert sind. Es sollte beachtet werden, dass jüngere im Vergleich zu älteren Personen alle Personalmarketingmaßnahmen im stärkeren Umfang nutzen und somit die in dieser Studie angegebenen aggregierten Werte der Nutzung möglicherweise etwas zu niedrig sind. Bezüglich der Bewertung der Personalmarketingmaßnahmen und der Bewerbungsrückmeldungen konnte das Alter hingegen als Kontrollvariable in die Analysen einbezogen werden, weshalb die Interpretierbarkeit der Ergebnisse hier gewährleistet ist. An dieser Stelle sei zudem angemerkt, dass die hier herangezogenen Daten mithilfe einer Online-Befragung erhoben wurden. Zum Erhebungszeitpunkt nutzen $97.6 \%$ der Berufstätigen in Deutschland das Internet (Frees \& Koch, 2018) und Online-Umfragen sind heutzutage etabliert, sodass der Effekt des Erhebungsmediums gering ausfallen dürfte.

\section{Praktische Implikationen}

Die vorliegende Studie liefert einen wichtigen Beitrag für die Entscheidungsgrundlage von Unternehmen und Organisationen in Bezug auf die Auswahl optimaler Recruitingstrategien. Aufgrund der gegenwärtigen Herausforderungen in der Personalbeschaffung (Weitzel et al., 2020a) ist es generell wichtig in die Optimierung der Recruitingstrategien zu investieren. Grundsätzlich ist Unternehmen und Organisationen zu empfehlen, weiterhin vorrangig etablierte Methoden des Recruitings zu nutzen 
- unabhängig vom Berufssektor oder Merkmalen der zu besetzenden Stelle. Diese Empfehlung lässt sich sowohl beim Personalmarketing als auch bei der Bewerbungsrückmeldung, nachdem eine Bewerbung eingegangen ist, aussprechen.

Beim Personalmarketing bietet es sich an, sowohl offline über persönliche Kontakte oder anhand von Stellenanzeigen in Zeitungen als auch online durch Stellenanzeigen auf der Unternehmenswebsite oder in Online-Jobportalen nach potenziellen Bewerber_innen zu suchen. Ergänzend können weitere Offline-Maßnahmen, wie der Kontakt zur Agentur für Arbeit, Kontakte zu Lehrern / Dozenten, (Inhouse) Bewerbertage, Karrieremessen, Firmenpräsentationen, Karrierebroschüren oder Firmenrankings eingesetzt werden. Da die meisten Unternehmen ohnehin schon mehr als einen Weg des Personalmarketings nutzen (Brenzel et al., 2016; Kubis, 2019), ist eine Kombination mehrerer Maßnahmen in vielen Fällen wahrscheinlich am effektivsten. So könnten klassische Maßnahmen, die von den Bewerber_innen präferiert werden, mit neuartigen Online-Maßnahmen, die für das Image eines Unternehmens zuträglich sind (Konradt \& Sarges, 2003), verknüpft werden.

Auch nachdem eine Bewerbung im Unternehmen eingegangen ist, sollten klassische Kommunikationswege genutzt werden - wie die in dieser Studie untersuchte Rückmeldung per Post. Dabei kann aber auch auf eine Rückmeldung per Telefon oder (zur Datensicherheit angemessen verschlüsselte) E-Mail zurückgegriffen werden - es ist anzunehmen, dass diese in ähnlich hohem Ausmaß bekannt und etabliert sind. Bei den neuartigen Technologien scheint es hingegen an Vertrauen seitens der Bewerber_innen zu fehlen, da diese unter anderem um ihre persönlichen Daten besorgt sind (Bohmova \& Malinová, 2013; Viseu, Clement, \& Aspinall, 2004). Unternehmen ist also zu empfehlen, die sehr neuartigen Methoden im Recruiting derzeit nicht zu verwenden solange derartige Bedenken bestehen. Auch wenn neuartige digitale Recruitingstrategien aus Sicht der Unternehmen attraktiv erscheinen, da beispielsweise Zeit und Kosten gespart werden können (Madia, 2011), sind es schlussendlich die Bewerber_innen, die über den Erfolg oder Misserfolg der Stellenbesetzung entscheiden (Chapman et al., 2005).

\section{Fazit}

Klassische Online- und Offline-Maßnahmen sind im Recruiting - sowohl beim Personalmarketing als auch bei der Bewerbungsrückmeldung - für alle Bewerbergruppen in puncto Nutzung und Bewertung führend, dabei findet sich ein Mere-Exposure-Effekt. Bemerkenswert ist, dass Online-Maßnahmen, die aufgrund ihrer Neuheit vor der Jahrtausendwende kaum Erwähnung fanden, wie beispielsweise Stellenanzeigen auf der Unternehmenswebsite oder in Online-Jobportalen (Breaugh, 2013), heutzutage nicht mehr aus dem Personalmarketing wegzudenken sind. Vielleicht begründet dies neben inhaltlichen Aspekten auch das steigende Interesse an neuartigen digitalen Methoden seitens der Unternehmen und der Fachöffentlichkeit (van Iddekinge et al., 2016; Weitzel et al., 2020a; Woods et al., 2020).

Neuartige digitale Web 2.0 Methoden wie die sozialen Medien werden jedoch aktuell von den Bewerber_innen wenig genutzt und als wenig ansprechend im Personalmarketing bewertet. Viele Autoren sind sich dennoch einig, dass die sozialen Medien großes Potenzial für zukünftiges Recruiting darstellen und einen starken Aufschwung erleben könnten (Chapman \& Gödöllei, 2017; McFarland \& Ployhart, 2015; Woods et al., 2020) - denn es sind viele Vorteile im Einsatz von neuartigen digitalen Methoden erkennbar, nicht nur für die Unternehmen, sondern auch für die Bewerber_innen (Cappelli, 2001; Konradt \& Sarges, 2003). Die Zukunft wird zeigen, ob sich der Trend, Recruiting zu digitalisieren, weiter verstärkt und sich auch die sozialen Medien und neuartige Technologien nach und nach im Bewerbungsprozess etablieren können.

\section{Literatur}

Allen, D. G., Mahto, R. V. \& Otondo, R. F. (2007). Web-based recruitment: Effects of information, organizational brand, and attitudes toward a Web site on applicant attraction. The Journal of applied psychology, 92(6), 1696-1708. https://doi.org/10. 1037/0021-9010.92.6.1696

Arnold, D., Hillerich-Sigg, A. \& Nolte, A. (2017). Fachkräftemangel: Reaktionen der Betriebe sowie Auswirkungen auf Investitionsentscheidungen und Wachstum. Studie im Auftrag des Bundesministeriums für Wirtschaft und Energie: Abschlussbericht. Mannheim: Zentrum für Europäische Wirtschaftsforschung (ZEW).

Batinic, B. \& Appel, M. (2009). Nutzung von Online-Bewerbungen aus Sicht von Bewerbern und Unternehmen. Zeitschrift für Personalpsychologie, 8(1), 14-23. https://doi.org/10.1026/ $1617-6391.8 .1 .14$

Bechmann, S., Dahms, V., Tschersich, N., Frei, M., Leber, U. \& Schwengler, B. (2012). Fachkräfte und unbesetzte Stellen in einer alternden Gesellschaft: Problemlagen und betriebliche Reaktionen, IAB-Forschungsbericht, 13/2012.

Beisch, N. \& Schäfer, C. (2020). Ergebnisse der ARD/ZDF-Onlinestudie 2020: Internetnutzung mit großer Dynamik: Medien, Kommunikation, Social Media. Media Perspektiven, 9, $462-$ 481.

Bohmova, L. \& Malinová, L. (2013). Facebook user's privacy in recruitment process. IDIMT, $159-168$. 
Bossler, M., Kubis, A. \& Moczall, A. (2017). Neueinstellungen im Jahr 2016: Große Betriebe haben im Wettbewerb um Fachkräfte oft die Nase vorn. IAB-Kurzbericht, 18/2017.

Breaugh, J. A. (2008). Employee recruitment: Current knowledge and important areas for future research. Human Resource Management Review, 18(3), 103-118. https://doi.org/10.1016/j. hrmr.2008.07.003

Breaugh, J. A. (2013). Employee recruitment. Annual review of psychology, 64, 389 - 416. https://doi.org/10.1146/annurev-psych$113011-143757$

Brenzel, H., Czepek, J., Kubis, A., Moczall, A., Rebien, M., Röttger, C., Szameitat, J., Warning, A. \& Weber, E. (2016). Neueinstellungen im Jahr 2015: Stellen werden häufig über persönliche Kontakte besetzt. IAB-Kurzbericht, 04/2016.

Busemann, K. \& Gscheidle, C. (2010). Web 2.0: Nutzung steigt Interesse an aktiver Teilhabe sinkt. Media Perspektiven, (7-8), $359-368$.

Cappelli, P. (2001). Making the most of On-Line Recruiting. Harvard Business Review, 79, 139-146.

Carless, S. A. (2005). Person-job fit versus person-organization fit as predictors of organizational attraction and job acceptance intentions: A longitudinal study. Journal of Occupational and Organizational Psychology, 78, 411-429. https://doi.org/10. 1348/096317905X25995

Chapman, D. S. \& Gödöllei, A. F. (2017). E-Recruiting: Using technology to attract job applicants. In G. Hertel, D. Stone, R. Johnson \& J. Passmore (Hrsg.), The Wiley Blackwell Handbook of the Psychology of the Internet at Work (pp. 211-230), Hoboken: Wiley-Blackwell.

Chapman, D. S., Uggerslev, K. L., Carroll, S. A., Piasentin, K. A. \& Jones, D. A. (2005). Applicant attraction to organizations and job choice: A meta-analytic review of the correlates of recruiting outcomes. The Journal of applied psychology, 90(5), 928-944. https://doi.org/10.1037/0021-9010.90.5.928

Dettmann, E., Fackler, D., Müller, S., Neuschäffer, G., Slavtchev, V., Leber, U. \& Schwengler, B. (2019). Fehlende Fachkräfte in Deutschland: Unterschiede in den Betrieben und mögliche Erklärungsfaktoren: Ergebnisse aus dem IAB-Betriebspanel 2018. IAB-Forschungsbericht, 10/2019.

Dietz, M., Kubis, A., Leber, U., Müller, A. \& Stegmaier, J. (2013). Personalsuche in Deutschland: Kleine und mittlere Betriebe im Wettbewerb um Fachkräfte. IAB-Kurzbericht, 10/2013.

Frees, B. \& Koch, W. (2018). ARD/ZDF-Onlinestudie 2018: Zuwachs bei medialer Internetnutzung und Kommunikation. Media Perspektiven, 9, $398-413$.

Gade, C. D., Helfritz, K. H. \& Murmann, J. (2018). Recruiting zwischen Executive Search und Digitalisierung: Eine Kooperationsstudie von iubh, BDU und DGFP. IUBH Discussion Papers Business \& Management (No. 3/2018). Bad Honnef.

Girard, A. \& Fallery, B. (2011). e-Recruitment: From TransactionBased Practices to Relationship-Based Approaches. In T. Bondarouk, H. Ruël \& J. Looise (Hrsg.) Electronic HRM in Theory and Practice (pp. 143-158). Bingley: Emerald Group Publishing. https://doi.org/10.1108/S1877-6361(2011)0000008012

Göritz, A. S. \& Moser, K. (2002). Personalmarketing im Internet: Eine Untersuchung des Auftritts der 100 größten deutschen Unternehmen. Zeitschrift für Personalpsychologie, 3, 141-148.

Harborth, D. \& Pape, S. (2018). German Translation of the Concerns for Information Privacy (CFIP) Construct. Verfügbar unter SSRN 3112207: http://dx.doi.org/10.2139/ssrn.3112207

Hassenzahl, M., Platz, A., Burmester, M. \& Lehner, K. (2000, April). Hedonic and ergonomic quality aspects determine a software's appeal. In Proceedings of the SIGCHI conference on Human Factors in Computing Systems (pp. 201 -208). https://doi.org/ $10.1145 / 332040.332432$
Hausknecht, J. P., Day, D. V. \& Thomas, S. C. (2004). Applicant Reactions to Selection Procedures: An Updated Model and Meta-Analysis. Personnel Psychology, 57(3), 639-683. https:// doi.org/10.1111/j.1744-6570.2004.00003.x

Iddekinge, C. H. van, Lanivich, S. E., Roth, P. L. \& Junco, E. (2016). Social Media for Selection? Validity and Adverse Impact Potential of a Facebook-Based Assessment. Journal of Management, 42, 1811 - 1835. https://doi.org/10.1177/0149206313515524

Kanning, U. P. (2016). Wie Bewerberinnen und Bewerber die Praxis der Personalauswahl erleben und bewerten. Report Psychologie, 2, $56-66$.

Konradt, U. \& Sarges, W. (2003). Suche, Auswahl und Förderung von Personal mit dem Intra-und Internet: Strategien, Zielrichtungen und Entwicklungspfade. E-Recruitment und E-Assessment, 3-16.

Kubis, A. (2019). IAB-Stellenerhebung 1/2019: Weiterhin hoher Bestand an offenen Stellen. In IAB-Forum. Verfügbar unter https://www.iab-forum.de/iab-stellenerhebung-1 - 2019-wei terhin-hoher-bestand-an-offenen-stellen/

Langer, M., König, C. J. \& Krause, K. (2017). Examining digital interviews for personnel selection: Applicant reactions and interviewer ratings. International Journal of Selection and Assessment, 25(4), 371 - 382. https://doi.org/10.1111/ijsa.12191

Langer, M., König, C. J. \& Papathanasiou, M. (2019). Highly automated job interviews: Acceptance under the influence of stakes. International Journal of Selection and Assessment, 27, 217-234. https://doi.org/10.1111/ijsa.12246

Lievens, F. \& Slaughter, J. E. (2016). Employer Image and Employer Branding: What We Know and What We Need to Know. Annual Review of Organizational Psychology and Organizational Behavior, 3, 407 - 440. https://doi.org/10.1146/annurev-orgpsych$041015-062501$

Lyons, B. D. \& Marler, J. H. (2011). Got image? Examining organizational image in web recruitment. Journal of Managerial Psychology, 26(1), 58-76. https://doi.org/10.1108/ 02683941111099628

Madia, S. A. (2011). Best practices for using social media as a recruitment strategy. Strategic HR Review, 10(6), 19-24. https://doi.org/10.1108/14754391111172788

Matthes, B., Meinken, H. \& Neuhauser, P. (2015). Berufssektoren und Berufssegmente auf Grundlage der KldB 2010. Methodenbericht der Statistik der BA. Nürnberg.

McFarland, L. A. \& Ployhart, R. E. (2015). Social media: A contextual framework to guide research and practice. The Journal of applied psychology, 100, 1653-1677. https://doi.org/10.1037/ a0039244

Reinecke, L. \& Trepte, S. (2008). Privatsphäre 2.0: Konzepte von Privatheit, Intimsphäre und Werten im Umgang mit „user-generated-content. Kommunikation, Partizipation und Wirkungen im Social Web, 1, $205-228$.

Seng, A., Fiesel, L. \& Krol, B. (2012). Erfolgreiche Wege der Rekrutierung in Social Networks (KCS Schriftenreihe, Bd 4). Essen: MA Akademie Verlags-und Druck-Gesellschaft mbH

Smith, H. J., Milberg, S. J. \& Burke, S. J. (1996). Information Privacy: Measuring Individuals' Concerns about Organizational Practices. MIS Quarterly, 20(2), 167. https://doi.org/10.2307/ 249477

Statistisches Bundesamt (2019). Bevölkerung und Erwerbstätigkeit: Erwerbsbeteiligung der Bevölkerung - Ergebnisse des Mikrozensus zum Arbeitsmarkt 2018 (Fachserie 1, Reihe 4.1). Wiesbaden: Statistisches Bundesamt.

Storrer, A. (2013). Sprachstil und Sprachvariation in sozialen Netzwerken. In B. Frank-Job, A. Mehler \& T. Sutter (Hrsg.), Die Dynamik sozialer und sprachlicher Netzwerke (S. 331-366). Wiesbaden: Springer. https://doi.org/10.1007/978-3-53193336-8_15 
Taddicken, M. \& Schmidt, J.-H. (2017). Entwicklung und Verbreitung sozialer Medien. In J.-H. Schmidt \& M. Taddicken (Hrsg.), Handbuch Soziale Medien (S. 3-22). Wiesbaden: Springer. https://doi.org/10.1007/978-3-658-03765-9_1

Taylor, H. C. \& Russell, J. T. (1939). The relationship of validity coefficients to the practical effectiveness of tests in selection: Discussion and tables. The Journal of Applied Psychology, 23, 565 - 578. https://doi.org/10.1037/h0057079

Thielsch, M. T. Träumer, L. \& Pytlik, L. (2012a). E-Recruiting and fairness - the applicant's point of view. Information Technology and Management, 13(2), 59-67. https://doi.org/10.1007/ s10799-012-0117-x

Thielsch, M. T., Träumer, L., Pytlik, L. \& Kanning, U. P. (2012b). Personalmarketing aus Bewerbersicht: Nutzung und Bewertung. Journal of Business and Media Psychology, 3(1), 1 - 12.

Truxillo, D. M., Bauer, T. N. \& Garcia, A. M. (2017). Applicant reactions to hiring procedures. In W. Goldstein, E. D. Pulakos, J. Passmore \& C. Semedo (Hrsg.), The Wiley Blackwell Handbook of the Psychology of Recruitment, Selection and Employee Retention (pp. 53 - 70). Hoboken: Wiley-Blackwell.

Viseu, A., Clement, A. \& Aspinall, J. (2004). Situating Privacy Online. Information, Communication \& Society, 7(1), 92-114. https:// doi.org/10.1080/1369118042000208924

Weitzel, T., Maier, C., Weinert, C., Pflügner, K., Oehlhorn, C., Wirth, J. \& Laumer, S. (2020a). Employer Branding - Ausgewählte Ergebnisse der Recruiting Trends 2020 und der Bewerbungspraxis 2020. Bamberg: Otto-Friedrich-Universität Bamberg.

Weitzel, T., Maier, C., Weinert, C., Pflügner, K., Oehlhorn, C., Wirth, J. \& Laumer, S. (2020b). Social Recruiting und Active Sourcing Ausgewählte Ergebnisse der Recruiting Trends 2020 und der Bewerbungspraxis 2020. Bamberg: Otto-Friedrich-Universität Bamberg.

Weitzel, T., Eckhardt, A., Laumer, S., Stetten, A. von, Maier, C. \& Weinert, C. (2015). Recruiting Trends 2014: Eine empirische Untersuchung mit den Top-1.000-Unternehmen aus Deutschland sowie den Top-300-Unternehmen aus den Branchen Health Care, IT und Maschinenbau. Bamberg: Otto-Friedrich-Universität Bamberg.

Weitzel, T., Laumer, S., Maier, C., Oehlhorn, C., Wirth, J., Weinert, C. \& Eckhardt, A. (2016). Bewerbung der Zukunft: Ausgewählte Ergebnisse der Recruiting Trends 2016. Bamberg: Otto-Friedrich-Universität Bamberg.

Woods, S. A., Ahmed, S., Nikolaou, I., Costa, A. C. \& Anderson, N. R. (2020). Personnel selection in the digital age: A review of validity and applicant reactions, and future research challenges. Euro- pean Journal of Work and Organizational Psychology, 29(1), 64-77. https://doi.org/10.1080/1359432X.2019.1681401

Yao, M. Z., Rice, R. E. \& Wallis, K. (2007). Predicting user concerns about online privacy. Journal of the American Society for Information Science and Technology, 58, 710-722. https://doi.org/ 10.1002/asi.20530

Zajonc, R. (1968). Attitudinal Effects of Mere Exposure. Journal of Personality and Social Psychology, 9, 1-27. https://doi.org/10. 1037/h0025848

\section{Historie}

Eingegangen: 01.07.2020

Revision eingegangen: 02.02.2021

Onlineveröffentlichung: 19.04. 2021

\section{Danksagung}

Die Autoren möchten sich herzlich bei Dr. Otto Hellwig und der respondi AG für die Unterstützung der Studie sowie bei Dominik Sondern für das Feedback zu früheren Manuskriptversionen bedanken! Die Autoren danken zudem den anonymen Reviewern für ihre sehr hilfreichen Kommentare.

\section{Open Data}

Das Online-Supplement inkl. anonymisierter Rohdaten findet sich unter https://doi.org/10.5281/zenodo.4520980

\section{Förderung}

Open Access-Veröffentlichung ermöglicht durch die Westfälische Wilhelms-Universität Münster

\section{ORCID}

Meinald T. Thielsch

(D)https://orcid.org/0000-0001-8493-9071

Viola Merhof

(D)https://orcid.org/0000-0002-1328-0000

\section{Prof. Dr. Meinald T. Thielsch}

Institut für Psychologie

Universität Münster

Fliednerstraße 21

48149 Münster

thielsch@uni-muenster.de 